\title{
Review Article \\ Targeting Inflammation in Emerging Therapies for Genetic Retinal Disease
}

\author{
Ishaq A. Viringipurampeer, Abu E. Bashar, Cheryl Y. Gregory-Evans, \\ Orson L. Moritz, and Kevin Gregory-Evans
} Eye Care Centre, Department of Ophthalmology and Visual Science, University of British Columbia,
2550 Willow Street, Vancouver, BC, Canada V5Z 3N9

Correspondence should be addressed to Kevin Gregory-Evans; kge30@eyecarecentre.org

Received 5 November 2012; Accepted 20 January 2013

Academic Editor: David A. Hollander

Copyright (c) 2013 Ishaq A. Viringipurampeer et al. This is an open access article distributed under the Creative Commons Attribution License, which permits unrestricted use, distribution, and reproduction in any medium, provided the original work is properly cited.

Genetic retinal diseases such as age-related macular degeneration and monogenic diseases such as retinitis pigmentosa account for some of the commonest causes of blindness in the developed world. Diverse genetic abnormalities and environmental causes have been implicated in triggering multiple pathological mechanisms such as oxidative stress, lipofuscin deposits, neovascularisation, and programmed cell death. In recent years, inflammation has also been highlighted although whether inflammatory mediators play a central role in pathogenesis or a more minor secondary role has yet to be established. Despite this, numerous interventional studies, particularly targeting the complement system, are underway with the promise of novel therapeutic strategies for these important blinding conditions.

\section{Introduction}

Inherited retinal diseases include some of the commonest causes of blindness in the developed world $[1,2]$. Prominent examples included age-related macular degeneration (AMD), diabetic retinopathy, and the numerous monogenic conditions such as retinitis pigmentosa (RP), Stargardt's disease, and X-linked retinoschisis. As well as causative/predisposing genetic abnormalities [3], in some cases (such as AMD), environmental factors such as smoking and diet have been highlighted $[4,5]$. These aetiological factors have been associated with diverse abnormal biochemical pathways in the degenerating retina, for instance, oxidative stress [6]; lipofuscin accumulation in the retinal pigment epithelium [7]; abnormalities of the extracellular matrix [8]; mitochondrial abnormalities [9]; ischaemia with neovascularisation [10]; programmed cell death [11]. In particular in AMD and $\mathrm{RP}$, inflammation has recently become a prominent member of this list of abnormal pathological pathways triggered by genetic retinal disease $[12,13]$.

\section{Established Links between Inflammation and Genetic Retinal Disease}

Early studies showed that autoantibodies can be detected in blood of AMD patients $[14,15]$ and that macrophages also accumulate in the choroid [16] which suggested that immune-mediated processes were involved in the pathogenesis of AMD. Renewed interest in inflammation in genetic retinal disease was, however, more recently triggered by the discovery of elements of the immune system and multiple proteins of the complement pathway within the drusen seen in AMD [17]. Although the exact pathophysiology of AMD remains largely unknown, accumulation of drusen is acknowledged as an early and major pathological hallmark of the disease, preempting damage in the retinal pigment epithelium, photoreceptors, and choroid leading to atrophic or neovascular complications.

Immunohistochemical studies of human retina highlighted that amongst other components, AMD drusen contained inflammatory mediators such as vitronectin, 
immunoglobulin light chains, factor $\mathrm{X}$, and complement proteins (C5 and C5b-9 complex). Importantly, it was also demonstrated that drusen displays intense HLA-DR immunoreactivity $[17,18]$. This later finding complements other independent work suggesting and association between AMD and HLA-genotype [19]. It is, however, now recognised that the composition of drusen deposits is extremely heterogeneous amongst different patients and that many other components are also found in addition to inflammatory mediators. For instance, other studies have highlighted the appearance of oxidative protein modifications within AMD drusen including cross-linked species of tissue metalloproteinase inhibitor 3, vitronectin, and carboxyethyl pyrrole protein adducts suggesting oxidative stress as an etiological factor in AMD drusen formation [20].

The feasibility of linking immunity to AMD pathophysiology has also been suggested by the central role the retinal pigment epithelium (RPE) plays in both AMD pathogenesis [21] and ocular immune modulation [22]. Both in vivo and in vitro experiments have demonstrated that the RPE expresses both innate and adaptive immune receptors [22, 23]. In addition, RPE cells are known to secrete numerous cytokines, chemokines, and adhesive molecules including interleukin6 , interleukin-8, and immunosuppressive factors including tissue-necrosis factor- $\beta$, interleukin-11, and interferon- $\beta$ [22].

Evidence suggesting a role for inflammation in AMD has, however, been strongly supported by molecular genetic studies. In particular, genes encoding components of the complement pathways have been associated with AMD. Strong associations have been demonstrated for alleles of genes encoding complement factor $\mathrm{H}(\mathrm{CFH})$ [24-26] which is a regulator of the alternate complement pathway, complement component $\mathrm{C} 2 /$ complement factor B (C2/CFB) [27], and CFH-related genes CFHR1 and CFHR3 [28]. Weaker associations have been linked to complement factor 1; complement C3 and complement component 1 inhibitor (SERPING1), a regulator of the classic complement pathway; chemokine C-X3-C receptor $1(C X 3 C r 1)$; and toll-like receptor genes TLR3 and TLR4 (with a role in the innate immune system) $[29,30]$. It should be noted, however, that strong genetic associations between loci and AMD also exist, for example, to the PLEKHA1/ARMS2/HTRA1 region of chromosome 10q26 [29].

Most recently, however, there has been remarkable work linking nucleotide-binding domain and leucine-rich-repeatprotein 3 (NLRP3) and the "inflammasome" with the aetiology of ARMD [31-33]. The inflammasome is a term used to identify a collection of proteins that work together within cells with a common purpose, more specifically, a caspase1 dependant multiprotein complex that has a key role in innate immunity. The inflammasome can be triggered by a number of stimuli including microbial pathogen-associated molecular patterns, bacterial toxins and most relevant to genetic retinal disease "damage-associated molecular patterns" (denatured nuclear or cytosolic proteins released from dying cells). This results in the upregulation of proinflammatory cytokines interleukin- $1 \beta$ and interleukin-18 $[34,35]$. The inflammasome can be activated by three classes of immune sensors including the toll-like receptors, RIG-I-like helicases, and NLR proteins [36]. Currently, four inflammasomes based on NLRP3 have been characterised: NLRP1/NLRP1b [37]; NLRC4/IPAF [38]; NLRP3/NALP3 [39]; AIM2 [40]. Activation of the NLRP3 inflammasome has recently been reported in dry AMD drusen, by complement component C1Q or by carboxyethylpyrrole (during oxidative stress) [31]. In wet ARMD, activation of the NLRP3 inflammasome has been seen triggered by Alu RNA molecules (short chains RNA) [32]. Intriguingly, reflecting the diversity of immune responses, the most recent in vivo and in vitro studies have suggested that the NLRP3 inflammasomes may have a beneficial role in wet ARMD but a harmful effect leading to RPE cell death in dry AMD $[31,32]$.

Linking inflammatory mediators to the choroidal neovascularization seen in complicated AMD is also firmly established. In both preclinical and clinical studies, cellular components of immunity including macrophages, lymphocytes and neutrophils have been found to be significant components of choroidal neovascular complexes [16, 41, 42]. Additionally, inflammatory cytokines such as interleukin-6 and interleukin- 8 have also been identified in the aqueous humor of AMD patients suffering from choroidal neovascularization [43].

While the term "retinitis pigmentosa" as coined by Donders in 1857 is generally considered a misnomer, a role for inflammation and immunity in the pathogenesis of the disease has significant merit. The earliest studies to suggest this showed elevated IgM in six out of ten RP patients [44]. Other early studies also suggested that retinal (probably photoreceptor) autoantibodies could be found in the systemic circulation of RP patients [45-48]. Immune reactivity in $\mathrm{RP}$ was also established by exposing lymphoctyes and leuckocytes from blood samples of RP patients to human and bovine retinal antigens [49]. However, results have been complicated by the fact that immune reactivity appears to vary amongst RP patients possibly reflecting the genetic heterogeneity of the disease. Studies suggested that circulating immune complexes could be detected in less than $50 \%$ of RP patients [50]. This was, however, reported as significant since it correlated with statistically significant reductions of circulating complement factors $\mathrm{C} 3$ and $\mathrm{C} 4$ and a significant reduction in time taken for RP patient sera to achieve $50 \%$ hemolysis of sheep red blood cells [50]. A link with HLA status has also been reported in RP patients [51], and vitreous samples from RP patients have been shown to contain many immune system cells such as various types of lymphocytes [52].

More recent studies have highlighted the activation of microglia in RP retina preceding photoreceptor cell death. Activation of microglia results in many biochemical events including the release of cytokines and chemokines [53]. In $r d$ mice (a homozygous nonsense phosphodiesterase-beta subunit gene mutant), it has been shown that prior to peak photoreceptor cell death, there is an upregulation of mRNA of proinflammatory factors: monocyte chemoattractant proteins 1 and 3; macrophage inflammatory proteins lalpha and lbeta; regulated on activation normal T-cell expressed and secreted (RANTES); tumor necrosis factor-alpha [54]. Microglia activation and upregulation of proinflammatory 
markers has also been demonstrated to precede peak photoreceptor cell death in rd10 mice (homozygous missense phosphodiesterase beta subunit mutant) [55].

Although direct biochemical assessment of retina from human RP patients is difficult, more detailed recent studies have searched for signs of inflammatory cells and humoral inflammatory factors in aqueous and vitreous humor from RP patients [13]. Slit lamp examination revealed that cells could be visualised in the anterior chamber $37 \%$ (190) and the vitreous of $61 \%$ (313) of $509 \mathrm{RP}$ eyes (up to 30 cells identified in a $1 \times 9 \mathrm{~mm}$ vertical slit-lamp field). It was not explained however how it was concluded that these were inflammatory cells (and not for instance, pigmentary cells). The study did, however, also report multiplex ELISA data suggesting significantly increased protein levels of cytokines (interleukin 6) and chemokines (interleukin-8, monocyte chemoattractant protein 1 , and thymus activation-regulated chemokine) in the aqueous. Much more notably, in the vitreous of these RP patients, significantly elevated levels of cytokines (interleukin $1 \alpha, 1 \beta, 2,4,6$, and 10 , interferon- $\gamma$ and tissue necrosis factor- $\alpha$ ) along with chemokines (such as interleukin-8, monocyte chemoattractant proteins 1 and 2 , interferon- $\gamma$ inducible protein-10, and thymus activationregulated chemokine) were found [13].

\section{Therapeutic Targets}

Numerous therapeutic strategies are emerging in the treatment of genetic retinal disease. These include cell-based therapies [56]; gene therapy [57]; electronic retinal replacements [58]; molecular-based approaches such as neuroprotection [59] and antiangiogenesis [60]. With an established role for inflammatory mediators in the pathogenesis of both AMD and RP, it would therefore be rational to investigate anti-inflammatory approaches. However, despite numerous animal models for RP [61], no universally recognised animal model for AMD yet exists, and only approximations modeling aspects of the disease are available [62]. This has limited preclinical research into AMD treatment. In addition, it is as yet unclear what role relative to other pathogenic mechanisms inflammation plays in disease pathogenesis. Anti-inflammatory approaches, for example, might have little impact on disease if inflammation is a secondary effect or a minor contributor to pathogenesis. To some extent, therefore, the importance of inflammation in AMD and RP can be validated through quantification of the effect of antiinflammatory therapeutics.

There are currently 846 listed clinical trials (http://www .clinicaltrials.gov/) focused on AMD of which 51 are specifically targeting inflammation. There are also 78 clinical trials listed for RP, although none are focused on anti-inflammatory therapeutics. Studies targeting inflammation in AMD and RP may be subdivided into broad approaches targeting multiple components of inflammation or more specific targeting of complement activation, for instance.

3.1. Broad-Based Anti-Inflammatory Studies in AMD. Corticosteroids have been used in retinal disease for many years because of their general anti-inflammatory effect, generally up-regulating the expression of anti-inflammatory proteins and suppressing the expression of proinflammatory factors $[63,64]$. Although their use in uveitis and retinitis is well established, corticosteroids are now being considered in AMD. Many studies have looked at the use of intravitreal dexamethasone in the treatment of neovascular complications of AMD, mostly as an adjuvant therapy in combination with photodynamic therapy, intravitreal anti-VEGF therapy, or in multitherapy approaches $[65,66]$. A sustained release dexamethasone (such as Ozurdex, Allergan, Inc., Irvine, CA) would seem to be the logical option in such an adjuvant approach. One single-masked, randomized control study in 243 eyes reported work comparing intravitreal ranibizumab plus sustained release dexamethasone with ranibizumab plus sham. Results suggested a reduction in the need for multiple ranibizumab injections and an increasing interval between injections although combination therapy was associated with raised intraocular pressure requiring treatment in $16 \%$ [67, 68]. A number of other clinical trials are now assessing dexamethasone as an adjunct in the treatment of wet AMD, and full reports are awaited (http://www.clinicaltrials.gov/, NCT01162746; NCT00793923; NCT00390208).

Other approaches include Iluvien, an intravitreal implant containing fluocinolone acetonide packed in a nonbiodegradable polyamide tube [69]. An ongoing phase 2 clinical trial is currently recruiting patients to look at the efficacy of this intravitreal implant in inhibiting geographic atrophy progression in AMD (http://www.clinicaltrials.gov/, NCT00695318).

Rapamycin, a drug originally designed as an antifungal agent, has recently been shown to be a potent immunosuppressive, anti-inflammatory, and antiangiogenesis agent by inhibiting the mammalian target of rapamycin (mTOR), a serine/threonine protein kinase. Recent work in the senescence-accelerated OXYS rat has shown some inhibition of the spontaneous retinopathy phenotype seen which models age-related macular degeneration in some respects [70]. A phase 2 study has been launched by National Eye Institute to determine whether repeated intravitreal rapamycin can slow progression of geographic atrophy (http://www.clinicaltrials.gov/, NCT01445548). Glatiramer acetate is another broad immunomodulatory agent that upregulates specific suppressor T-cells and suppresses inflammatory cytokines. Intravenous glatiramer acetate in dry AMD patients has been shown to reduce drusen load on optical coherence tomography imaging [71]. In the "wet" form of AMD, anti-inflammatory agents have been found to be moderately successful in controlling choroidal neovascularization. For example, intravitreal triamcinolone acetonide and infliximab, an antibody of tumor necrosis factor $\alpha$ (TNF$\alpha$ ), have shown positive effects in treating CNV in patients and animal models $[72,73]$.

\subsection{Focused Targeting of the Complement System in AMD.} The area in which inflammatory mediators in genetic retinal disease is being most intensively investigated, however, is in inhibiting activation of the complement system in AMD patients [74]. These studies have focused on both 
antibody dependent and independent complement inhibition trials.

FCFD4514S is a recombinant, humanized monoclonal antibody Fab fragment antibody targeted to block the complement factor $\mathrm{D}$, an early rate-limiting enzyme in the activation of the alternative complement pathway. Current phase 2 clinical trials are assessing its use in geographic atrophy (http://www.clinicaltrials.gov/, NCT01602120). Eculizumab (Soliris) is a humanized complement factor 5 monoclonal antibody that binds to complement factor 5 to block subsequent downstream anaphylatoxin activation and the formation of membrane attack complexes. It is considered by some to be the most likely approach since it preserves production of $\mathrm{C} 3 \mathrm{a}$ anaphylatoxin and $\mathrm{C} 3 \mathrm{~b}$ production required for opsonisation and clearing of harmful immune complexes [64]. Intravenous eculizumab is currently under trial for dry AMD (http://www.clinicaltrials.gov/, NCT00935883).

An example of studies focusing on nonantibody approaches to modify complement activation is POT-4, the first complement inhibitor to be assessed in AMD. This molecule is a cyclic peptide that reversibly binds to C3 preventing its activation to $\mathrm{C} 3 \mathrm{a}$ and $\mathrm{C} 3 \mathrm{~b}$, thus blocking the classic pathway and the lecithin pathway as well as the alternative complement pathway. Intravitreal depot injections are being assessed in patients with wet and dry AMD (http://www.clinicaltrials.gov/, NCT00473928).

Other nonantibody approaches include small molecule peptidomimetic C5a receptor antagonists currently being considered for AMD [75]. An alternative approach that may avoid the general consequences of inhibiting complement activation is studies focusing on replacing abnormal complement factor $\mathrm{H}$ alleles. TT30 is a recombinant fusion protein being used to replace defective complement factor $\mathrm{H}$ [76] and is being considered for use in AMD trials. Modification of complement activation may also be of benefit in wet AMD. In a laser-induced mouse model of choroidal neovascularization, intravenous administration of "CR2-fH," a recombinant form of complement factor $\mathrm{H}$ linked to complement receptor 2, inhibited neovascular growth [77].

3.3. Broad-Based Anti-Inflammatory Studies in RP. Corticosteroids have been routinely used to treat the macular edema seen in late RP with mixed success [78]. Most recently, however, a sustained-release dexamethasone implant (Ozurdex) has been used in a small cohort of RP patients with macular oedema, suggesting some structural and functional benefits [79]. Also recently, $N$-acetylcysteine, an orally bioavailable antioxidant, has been used in $r d 10$ mice [55]. It was shown by TUNEL staining that a reduction in photoreceptor cell death was associated with a strong suppression of expression of cytokines interleukin $1 \beta$ and tissue necrosis factor$\alpha$ and chemokines monocyte chemoattractant proteins 1 and thymus activation-regulated chemokine [55]. In another study, fluocinolone acetonide has been conjugated with dendrimer particles (a hydroxyl-terminated polyamidoamine dendrimer-drug conjugate nanodevice) to target outer retina activated microglia [80]. They showed that after intravitreal administration in the Royal College of Surgeons rat model of RP (homozygous Mertk mutant), four weeks later, there had been significant preservation of outer nuclear layer thickness (indicative of photoreceptor survival) and in electroretinogram b-wave response. In addition, it was shown that this was associated with a reduction of activated microglia in the retina [80].

\section{Conclusion}

Considerable evidence now exists linking inflammatory mediators to genetic retinal diseases such as AMD and RP. It is, however, still unclear whether this is a central or pivotal role [81]. Preclinical and clinical trials suggest that inhibiting inflammatory mediators can have some therapeutic benefit, but further ongoing trials are needed to demonstrate the true impact of this approach. The benefits of inhibiting inflammation in genetic retinal disease might, for instance not be so clear-cut. In recent clinical trials, ciliary-derived neurotrophic factor (CNTF, a neuroprotective growth factor) has been shown to provide some inhibition of degeneration in both dry AMD [82] and RP [83]. Studies in mouse retina, however, have suggested that CNTF induces expression of proinflammatory genes in retinal Müller cells [84]. This would seem counterintuitive, and its clinical relevance has yet to be determined. A role for anti-inflammatory agents, as stand-alone monotherapies or as adjuvants (for instance, in combination with neuroprotective strategies or anti-VEGF therapies), is certain to be prominent feature of future research into the treatment of retinal disease.

\section{Conflict of Interests}

There is no conflict of interests related to this paper.

\section{Acknowledgment}

This work was supported by funding from the Canadian Institutes of Health Research (Team Grant no. 222728).

\section{References}

[1] D. Pascolini and S. P. Mariotti, "Global estimates of visual impairment 2010," British Journal of Ophthalmology, vol. 96, no. 5, pp. 614-618, 2010.

[2] S. Resnikoff, D. Pascolini, D. Etyaale et al., "Global data on visual impairment in the year 2002," Bulletin of the World Health Organization, vol. 82, no. 11, pp. 844-851, 2004.

[3] A. F. Wright, C. F. Chakarova, M. M. Abd El-Aziz, and S. S. Bhattacharya, "Photoreceptor degeneration: genetic and mechanistic dissection of a complex trait," Nature Reviews Genetics, vol. 11, no. 4, pp. 273-284, 2010.

[4] S. C. Tomany, J. J. Wang, R. Van Leeuwen et al., "Risk factors for incident age-related macular degeneration: pooled findings from 3 continents," Ophthalmology, vol. 111, no. 7, pp. 1280-1287, 2004.

[5] J. S. L. Tan, J. J. Wang, V. Flood, E. Rochtchina, W. Smith, and P. Mitchell, "Dietary antioxidants and the long-term incidence of age-related macular degeneration: the Blue Mountains Eye Study," Ophthalmology, vol. 115, no. 2, pp. 334-341, 2008. 
[6] S. M. Plafker, G. B. O’Mealey, and L. I. Szweda, "Mechanisms for countering oxidative stress and damage in retinal pigment epithelium," International Review of Cell and Molecular Biology, vol. 298, pp. 135-177, 2012.

[7] N. P. Boyer, D. Higbee, M. B. Currin et al., "Lipofuscin and $N$-retinylidene- $N$-retinylethanolamine (A2E) accumulate in retinal pigment epithelium in absence of light exposure: their origin is 11-cis-retinal," Journal of Biological Chemistry, vol. 287, no. 26, pp. 22276-22286, 2012.

[8] J. C. Booij, D. C. Baas, J. Beisekeeva, T. G. M. F. Gorgels, and A. A. B. Bergen, "The dynamic nature of Bruch's membrane," Progress in Retinal and Eye Research, vol. 29, no. 1, pp. 1-18, 2010.

[9] A. Swaroop, E. Y. Chew, C. B. Rickman, and G. R. Abecasis, "Unraveling a multifactorial late-onset disease: from genetic susceptibility to disease mechanisms for age-related macular degeneration," Annual Review of Genomics and Human Genetics, vol. 10, pp. 19-43, 2009.

[10] L. S. Lim, P. Mitchell, J. M. Seddon, F. G. Holz, and T. Y. Wong, "Age-related macular degeneration," The Lancet, vol. 379, no. 9827, pp. 1728-1738, 2012.

[11] F. Doonan, G. Groeger, and T. G. Cotter, "Preventing retinal apoptosis-is there a common therapeutic theme?" Experimental Cell Research, vol. 318, no. 11, pp. 1278-1284, 2012.

[12] K. M. Gehrs, D. H. Anderson, L. V. Johnson, and G. S. Hageman, "Age-related macular degeneration-emerging pathogenetic and therapeutic concepts," Annals of Medicine, vol. 38, no. 7, pp. 450-471, 2006.

[13] N. Yoshida, Y. Ikeda, S. Notomi et al., "Clinical evidence of sustained chronic inflammatory reaction in retinitis pigmentosa," Ophthalmology, vol. 120, no. 1, pp. 100-105, 2013.

[14] P. L. Penfold, J. M. Provis, J. H. Furby, P. A. Gatenby, and F. A. Billson, "Autoantibodies to retinal astrocytes associated with age-related macular degeneration," Graefe's Archive for Clinical and Experimental Ophthalmology, vol. 228, no. 3, pp. 270-274, 1990.

[15] D. H. Gurne, M. O. M. Tso, D. P. Edward, and H. Ripps, "Antiretinal antibodies in serum of patients with age-related macular degeneration," Ophthalmology, vol. 98, no. 5, pp. 602607, 1991.

[16] M. C. Killingsworth, J. P. Sarks, and S. H. Sarks, "Macrophages related to Bruch's membrane in age-related macular degeneration," Eye, vol. 4, no. 4, pp. 613-621, 1990.

[17] R. F. Mullins, S. R. Russell, D. H. Anderson, and G. S. Hageman, "Drusen associated with aging and age-related macular degeneration contain proteins common to extracellular deposits associated with atherosclerosis, elastosis, amyloidosis, and dense deposit disease," FASEB Journal, vol. 14, no. 7, pp. 835846, 2000.

[18] G. S. Hageman, P. J. Luthert, N. H. V. Chong, L. V. Johnson, D. H. Anderson, and R. F. Mullins, "An integrated hypothesis that considers drusen as biomarkers of immune-mediated processes at the RPE-Bruch's membrane interface in aging and age-related macular degeneration," Progress in Retinal and Eye Research, vol. 20, no. 6, pp. 705-732, 2001.

[19] S. V. Goverdhan, S. I. Khakoo, H. Gaston, X. Chen, and A. J. Lotery, "Age-related macular degeneration is associated with the HLA-cw*0701 genotype and the natural killer cell receptor AA haplotype," Investigative Ophthalmology and Visual Science, vol. 49, no. 11, pp. 5077-5082, 2008.

[20] J. W. Crabb, M. Miyagi, X. Gu et al., "Drusen proteome analysis: an approach to the etiology of age-related macular degeneration," Proceedings of the National Academy of Sciences of the United States of America, vol. 99, no. 23, pp. 14682-14687, 2002.

[21] I. Bhutto and G. Lutty, "Understanding age-related macular degeneration (AMD): relationships between the photoreceptor/retinal pigment epithelium/Bruch's membrane/choriocapillaris complex," Molecular Aspects of Medicine, vol. 33, no. 4, pp. 295-317, 2012.

[22] B. Detrick and J. J. Hooks, "Immune regulation in the retina," Immunologic Research, vol. 47, no. 1-3, pp. 153-161, 2010.

[23] J. R. Sparrow, D. Hicks, and C. P. Hamel, "The retinal pigment epithelium in health and disease," Current Molecular Medicine, vol. 10, no. 9, pp. 802-823, 2010.

[24] R. J. Klein, C. Zeiss, E. Y. Chew et al., "Complement factor H polymorphism in age-related macular degeneration," Science, vol. 308, no. 5720, pp. 385-389, 2005.

[25] J. L. Haines, M. A. Hauser, S. Schmidt et al., "Complement factor $\mathrm{H}$ variant increases the risk of age-related macular degeneration," Science, vol. 308, no. 5720, pp. 419-421, 2005.

[26] A. O. Edwards, R. Ritter III, K. J. Abel, A. Manning, C. Panhuysen, and L. A. Farrer, "Complement factor $\mathrm{H}$ polymorphism and age-related macular degeneration," Science, vol. 308, no. 5720, pp. 421-424, 2005.

[27] B. Gold, J. E. Merriam, J. Zernant et al., "Variation in factor B $(\mathrm{BF})$ and complement component $2(\mathrm{C} 2)$ genes is associated with age-related macular degeneration," Nature Genetics, vol. 38, no. 4, pp. 458-462, 2006.

[28] A. E. Hughes, N. Orr, H. Esfandiary, M. Diaz-Torres, T. Goodship, and U. Chakravarthy, "A common CFH haplotype, with deletion of CFHR1 and CFHR3, is associated with lower risk of age-related macular degeneration," Nature Genetics, vol. 38, no. 10, pp. 1173-1177, 2006.

[29] Y. Chen, M. Bedell, and K. Zhang, "Age-related macular degeneration: genetic and environmental factors of disease," Molecular Interventions, vol. 10, no. 5, pp. 271-281, 2010.

[30] A. M. Newman, N. B. Gallo, L. S. Hancox et al., "Systemslevel analysis of age-related macular degeneration reveals global biomarkers and phenotype-specific functional networks?" Genome Medicine, vol. 4, no. 2, p. 16, 2012.

[31] S. L. Doyle, M. Campbell, E. Ozaki E et al., "NLRP3 has a protective role in age-related macular degeneration through the induction of IL-18 by drusen components," Nature Medicine, vol. 18, no. 5, pp. 791-798, 2012.

[32] V. Tarallo, Y. Hirano, B. D. Gelfand et al., "DICER1 loss and Alu RNA induce age-related macular degeneration via the NLRP3 inflammasome and MyD88," Cell, vol. 149, no. 4, pp. 847-859, 2012.

[33] H. Kaneko, S. Dridi, V. Tarallo et al., "DICER1 deficit induces Alu RNA toxicity in age-related macular degeneration," Nature, vol. 471, no. 7338, pp. 325-332, 2011.

[34] C. A. Dinarello, "Interleukin-1 $\beta$, interleukin-18, and the interleukin-1 $\beta$ converting enzyme," Annals of the New York Academy of Sciences, vol. 856, pp. 1-11, 1998.

[35] F. Martinon, K. Burns, and J. Tschopp, “The inflammasome: a molecular platform triggering activation of inflammatory caspases and processing of proIL- $\beta$," Molecular Cell, vol. 10, no. 2, pp. 417-426, 2002.

[36] S. Akira, S. Uematsu, and O. Takeuchi, "Pathogen recognition and innate immunity," Cell, vol. 124, no. 4, pp. 783-801, 2006.

[37] E. D. Boyden and W. F. Dietrich, "Nalplb controls mouse macrophage susceptibility to anthrax lethal toxin," Nature Genetics, vol. 38, no. 2, pp. 240-244, 2006. 
[38] Y. Zhao, J. Yang J, J. Shi et al., "The NLRC4 inflammasome receptors for bacterial flagellin and type III secretion apparatus," Nature, vol. 477, no. 7366, pp. 596-600, 2011.

[39] F. Martinon, V. Pétrilli, A. Mayor, A. Tardivel, and J. Tschopp, "Gout-associated uric acid crystals activate the NALP3 inflammasome," Nature, vol. 440, no. 7081, pp. 237-241, 2006.

[40] T. Fernandes-Alnemri, J. W. Yu, P. Datta, J. Wu, and E. S. Alnemri, "AIM2 activates the inflammasome and cell death in response to cytoplasmic DNA," Nature, vol. 458, no. 7237, pp. 509-513, 2009.

[41] L. L. Jones and D. A. Vignali, "Molecular interactions within the IL-6/IL-12 cytokine/receptor superfamily," Immunology Research, vol. 52, pp. 5-14, 2011.

[42] T. Tanaka, M. Narazaki, and T. Kishimoto, "Therapeutic targeting of the interleukin-6 receptor," Annual Reviews in Pharmacologic Toxicolgy, vol. 52, pp. 199-219, 2012.

[43] H. Miao, Y. Tao, and X. X. Li, "Inflammatory cytokines in aqueous humor of patients with choroidal neovascularization," Molecular Vision, vol. 18, pp. 574-580, 2012.

[44] W. J. Fessel, "Serum protein disturbance in retinitis pigmentosa. And the association of retinitis pigmentosa with psychosis," American Journal of Ophthalmology, vol. 53, no. 4, pp. 640-642, 1962.

[45] A. H. Rahi, "Autoimmunity and the retina. I. Antigenic specificity of photoreceptor cells," British Journal of Ophthalmology, vol. 54, no. 7, pp. 441-444, 1970.

[46] A. H. Rahi, "Autoimmunity and the retina. II. Raised serum IgM levels in retinitis pigmentosa," British Journal of Ophthalmology, vol. 57, no. 12, pp. 904-909, 1973.

[47] D. J. Spalton, A. H. S. Rahi, and A. C. Bird, "Immunological studies in retinitis pigmentosa associated with retinal vascular leakage," British Journal of Ophthalmology, vol. 62, no. 3, pp. 183187, 1978.

[48] V. G. Wong, W. R. Green, T. Kuwabara, P. R. McMaster, and T. P. Cameron, "Homologous retinal outer segment immunization in primates: a clinical and histopathological study," Transactions of the American Ophthalmological Society, vol. 72, pp. 184-195, 1974.

[49] C. J. J. Brinkman, A. J. L. G. Pinckers, and R. M. Broekhuyse, "Immune reactivity to different retinal antigens in patients suffering from retinitis pigmentosa," Investigative Ophthalmology and Visual Science, vol. 19, no. 7, pp. 743-750, 1980.

[50] C. D. Heredia, J. Huguet, N. Cols, P. Engel, and P. A. GarciaCalderon, "Immune complexes in retinitis pigmentosa," British Journal of Ophthalmology, vol. 68, no. 11, pp. 811-814, 1984.

[51] B. Detrick, M. Rodrigues, C. C. Chan, M. O. Tso, and J. J. Hooks, "Expression of HLA-DR antigen on retinal pigment epithelial cells in retinitis pigmentosa," American Journal of Ophthalmology, vol. 101, no. 5, pp. 584-590, 1986.

[52] D. A. Newsome and R. G. Michels, "Detection of lymphocytes in the vitreous gel of patients with retinitis pigmentosa," American Journal of Ophthalmology, vol. 105, no. 6, pp. 596-602, 1988.

[53] N. Gupta, K. E. Brown, and A. H. Milam, "Activated microglia in human retinitis pigmentosa, late-onset retinal degeneration, and age-related macular degeneration," Experimental Eye Research, vol. 76, no. 4, pp. 463-471, 2003.

[54] H. Y. Zeng, X. A. Zhu, C. Zhang, L. P. Yang, L. M. Wu, and M. O. M. Tso, "Identification of sequential events and factors associated with microglial activation, migration, and cytotoxicity in retinal degeneration in rd mice," Investigative Ophthalmology and Visual Science, vol. 46, no. 8, pp. 2992-2999, 2005.
[55] N. Yoshida, Y. Ikeda, S. Notomi et al., "Laboratory evidence of sustained chronic inflammatory reaction in retinitis pigmentosa," Ophthalmology, vol. 120, no. 1, pp. e5-e12, 2013.

[56] R. A. Pearson, A. C. Barber, M. Rizzi et al., "Restoration of vision after transplantation of photoreceptors," Nature, vol. 485, no. 7396, pp. 99-103, 2012.

[57] A. M. Maguire, K. A. High, A. Auricchio et al., "Age-dependent effects of RPE65 gene therapy for Leber's congenital amaurosis: a phase 1 dose-escalation trial," The Lancet, vol. 374, no. 9701, pp. 1597-1605, 2009.

[58] A. Kusnyerik, U. Greppmaier, R. Wilke et al., "Positioning of electronic subretinal implants in blind retinitis pigmentosa patients through multimodal assessment of retinal structures," Investigative Ophthalmology and Visual Science, vol. 53, no. 7, pp. 3748-3755, 2012.

[59] D. Trifunovic, A. Sahaboglu, J. Kaur et al., "Neuroprotective strategies for the treatment of inherited photoreceptor degeneration," Current Molecular Medicine, vol. 12, no. 5, pp. 598-612, 2012.

[60] D. R. Lally, A. T. Gerstenblith, and C. D. Regillo, "Preferred therapies for neovascular age-related macular degeneration," Current Opinion in Ophthalmology, vol. 23, no. 3, pp. 182-188, 2012.

[61] E. L. Fletcher, A. I. Jobling, K. A. Vessey, C. Luu, R. H. Guymer, and P. N. Baird, "Animal models of retinal disease," Progress in Molecular Biology and Translational Science, vol. 100, pp. 211286, 2011.

[62] M. E. Pennesi, M. Neuringer, and R. J. Courtney, "Animal models of age related macular degeneration," Molecular Aspects of Medicine, vol. 33, no. 4, pp. 487-509, 2012.

[63] A. Sallam, S. R. Taylor, and S. Lightman, "Review and update of intraocular therapy in noninfectious uveitis," Current Opinion in Ophthalmology, vol. 22, no. 6, pp. 517-522, 2011.

[64] Z. Yehoshua, P. J. Rosenfeld, and T. A. Albini, "Current clinical trials in dry AMD and the definition of appropriate clinical outcome measures," Seminars in Ophthalmology, vol. 26, no. 3, pp. 167-180, 2011.

[65] D. Ehmann and R. García, "Triple therapy for neovascular age-related macular degeneration (verteporfin photodynamic therapy, intravitreal dexamethasone, and intravitreal bevacizumab)," Canadian Journal of Ophthalmology, vol. 45, no. 1, pp. 36-40, 2010.

[66] S. Sivaprasad, S. Patra, J. Dacosta et al., "A pilot study on the combination treatment of reduced-fluence photodynamic therapy, intravitreal ranibizumab, intravitreal dexamethasone and oral minocycline for neovascular age-related macular degeneration," Ophthalmologica, vol. 225, no. 4, pp. 200-206, 2011.

[67] N. J. S. London, A. Chiang, and J. A. Haller, “The dexamethasone drug delivery system: indications and evidence," Advances in Therapy, vol. 28, no. 5, pp. 351-366, 2011.

[68] B. Kupperman, "Safety and efficacy of dexamethasone intravitreal implant as adjunctive therapy to Lucentis in patients with choroidal neovascularization secondary to age-related macular degeneration," in Proceedings of the 33rd Annual Macular Society Meeting, Tucson, Ariz, USA, February 2010.

[69] F. E. Kane, J. Burdan, A. Cutino, and K. E. Green, "Iluvien: a new sustained delivery technology for posterior eye disease," Expert Opinion on Drug Delivery, vol. 5, no. 9, pp. 1039-1046, 2008.

[70] N. G. Kolosova, N. A. Muraleva, A. A. Zhdankina, N. A. Stefanova, A. Z. Fursova, and M. V. Blagosklonny, "Prevention of age-related macular degeneration-like retinopathy by 
rapamycin in rats," American Journal of Pathology, vol. 181, no. 2, pp. 472-477, 2012.

[71] G. Landa, R. B. Rosen, A. Patel et al., "Qualitative spectral OCT/SLO analysis of drusen change in dry age-related macular degeneration patients treated with copaxone," Journal of Ocular Pharmacology and Therapeutics, vol. 27, no. 1, pp. 77-82, 2011.

[72] R. S. Kadam, P. Tyagi, H. F. Edelhauser, and U. B. Kompella, "Influence of choroidal neovascularization and biodegradable polymeric particle size on transscleral sustained delivery of triamcinolone acetonide," International Journal of Pharmacology, vol. 434, pp. 140-147, 2012.

[73] J. L. Olson, R. J. Courtney, and N. Mandava, "Intravitreal infliximab and choroidal neovascularization in an animal model," Archives of Ophthalmology, vol. 125, no. 9, pp. 1221-1224, 2007.

[74] R. Troutbeck, S. Al-Qureshi, and R. H. Guymer, "Therapeutic targeting of the complement system in age-related macular degeneration: a review," Clinical Experimental Ophthalmology, vol. 40, no. 1, pp. 18-26, 2012.

[75] K. Schnatbaum, E. Locardi, D. Scharn et al., "Peptidomimetic C5a receptor antagonists with hydrophobic substitutions at the C-terminus: increased receptor specificity and in vivo activity," Bioorganic and Medicinal Chemistry Letters, vol. 16, no. 19, pp. 5088-5092, 2006.

[76] M. Fridkis-Hareli, M. Storek, I. Mazsaroff et al., "Design and development of TT30, a novel C3d-targeted C3/C5 convertase inhibitor for treatment of human complement alternative pathway-mediated diseases," Blood, vol. 118, no. 17, pp. 47054713, 2011.

[77] B. Rohrer, Q. Long, B. Coughlin et al., "A targeted inhibitor of the alternative complement pathway reduces angiogenesis in a mouse model of age-related macular degeneration," Investigative Ophthalmology and Visual Science, vol. 50, no. 7, pp. 30563064, 2009.

[78] H. Ozdemir, M. Karacorlu, and S. Karacorlu, "Intravitreal triamcinolone acetonide for treatment of cystoid macular oedema in patients with retinitis pigmentosa," Acta Ophthalmologica Scandinavica, vol. 83, no. 2, pp. 248-251, 2005.

[79] M. Srour, G. Querques, N. Leveziel et al., "Intravitreal dexamethasone implant (Ozurdex) for macular edema secondary to retinitis pigmentosa," Graefe's Archive for Clinical and Experimental Ophthalmology, 2012.

[80] R. Iezzi, B. R. Guru, I. V. Glybina, M. K. Mishra, A. Kennedy, and R. M. Kannan, "Dendrimer-based targeted intravitreal therapy for sustained attenuation of neuroinflammation in retinal degeneration," Biomaterials, vol. 33, pp. 979-988, 2012.

[81] M. A. Zarbin and P. J. Rosenfeld, "Pathway-based therapies for age-related macular degeneration: an integrated survey of emerging treatment alternatives," Retina, vol. 30, no. 9, pp. 1350$1367,2010$.

[82] W. Xue, R. I. Cojocaru, V. J. Dudley, M. Brooks, A. Swaroop, and V. P. Sarthy, "Ciliary neurotrophic factor induces genes associated with inflammation and gliosis in the retina: a gene profiling study of flow-sorted, müller cells," PLoS ONE, vol. 6, no. 5, Article ID e20326, 2011.

[83] K. Zhang, J. J. Hopkins, J. S. Heier et al., "Ciliary neurotrophic factor delivered by encapsulated cell intraocular implants for treatment of geographic atrophy in age-related macular degeneration," Proceedings of the National Academy of Sciences of the United States of America, vol. 108, no. 15, pp. 6241-6245, 2011.

[84] K. E. Talcott, K. Ratnam, S. M. Sundquist et al., "Longitudinal study of cone photoreceptors during retinal degeneration and in response to ciliary neurotrophic factor treatment," Investigative Ophthalmology and Visual Science, vol. 52, no. 5, pp. 2219-2226, 2011. 


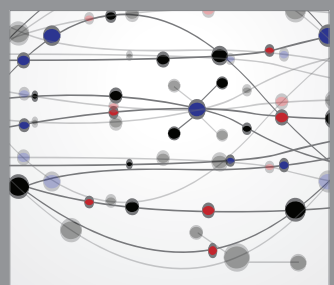

The Scientific World Journal
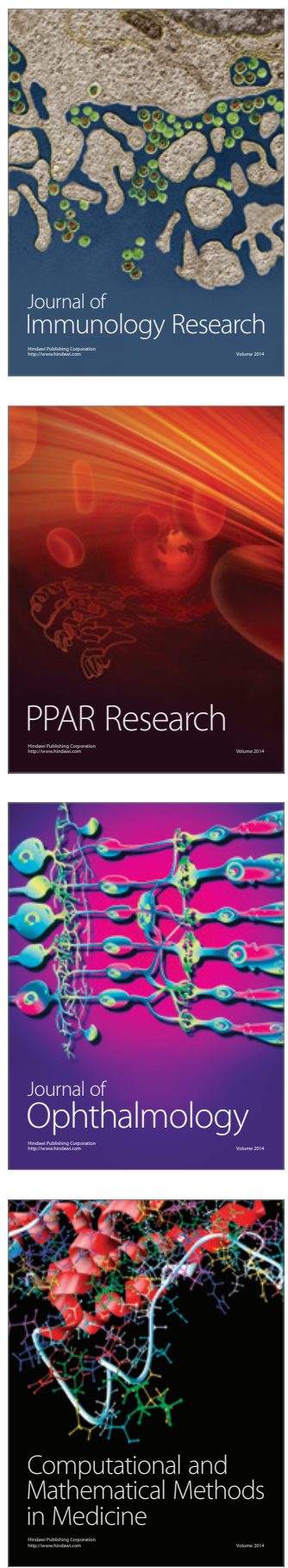

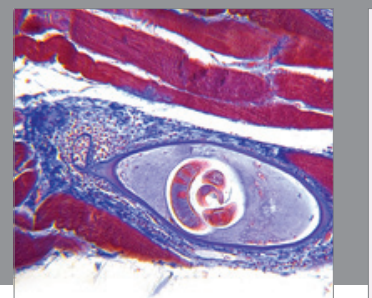

Gastroenterology

Research and Practice
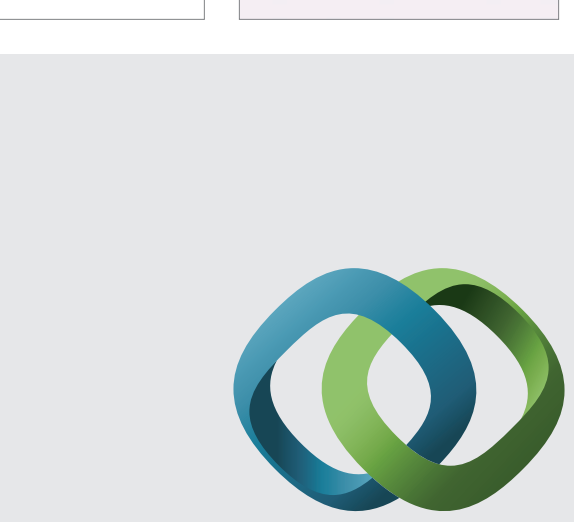

\section{Hindawi}

Submit your manuscripts at

http://www.hindawi.com
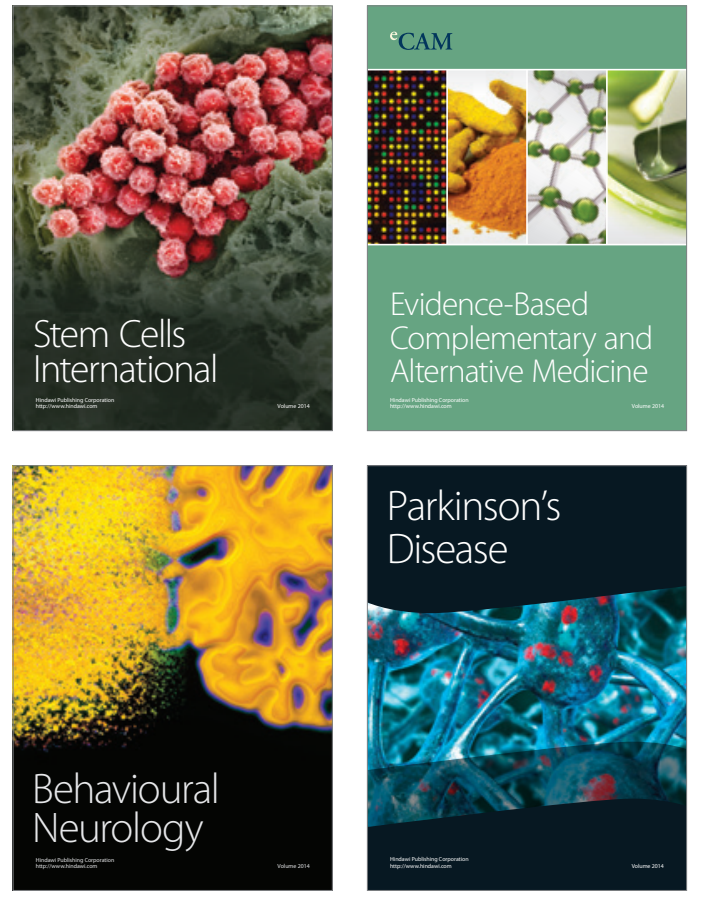
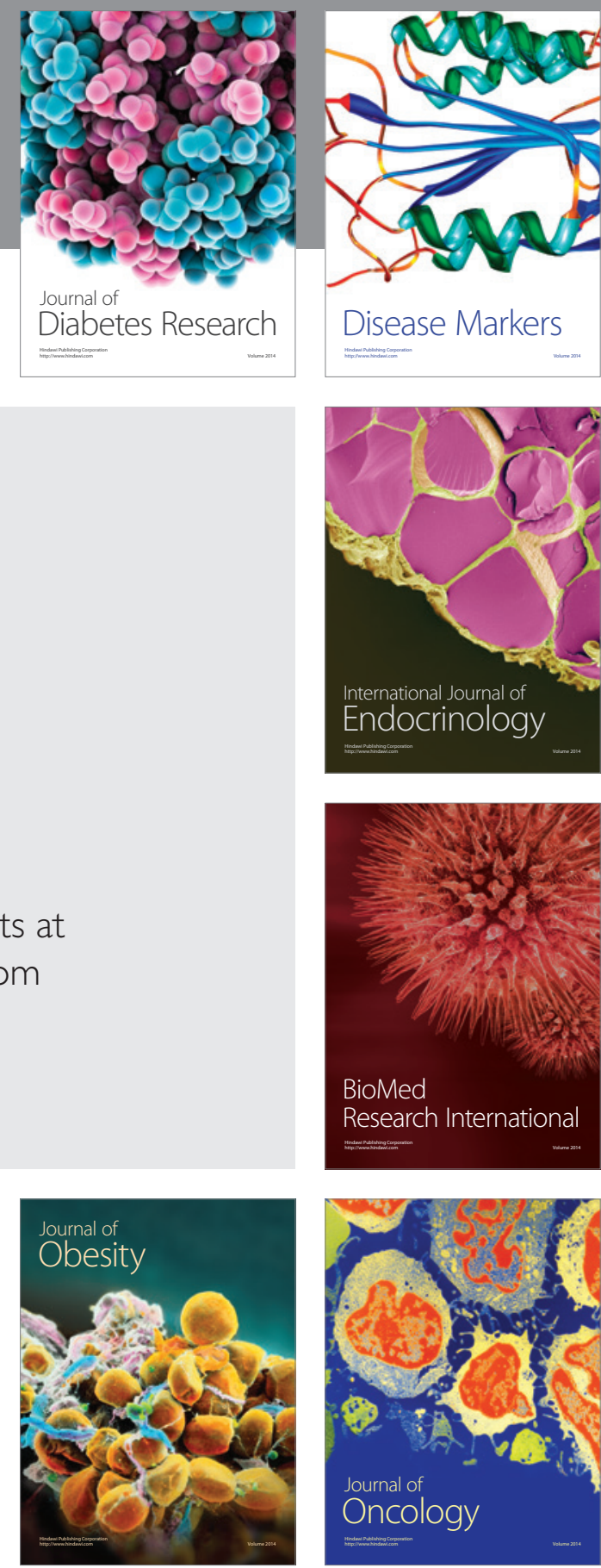

Disease Markers
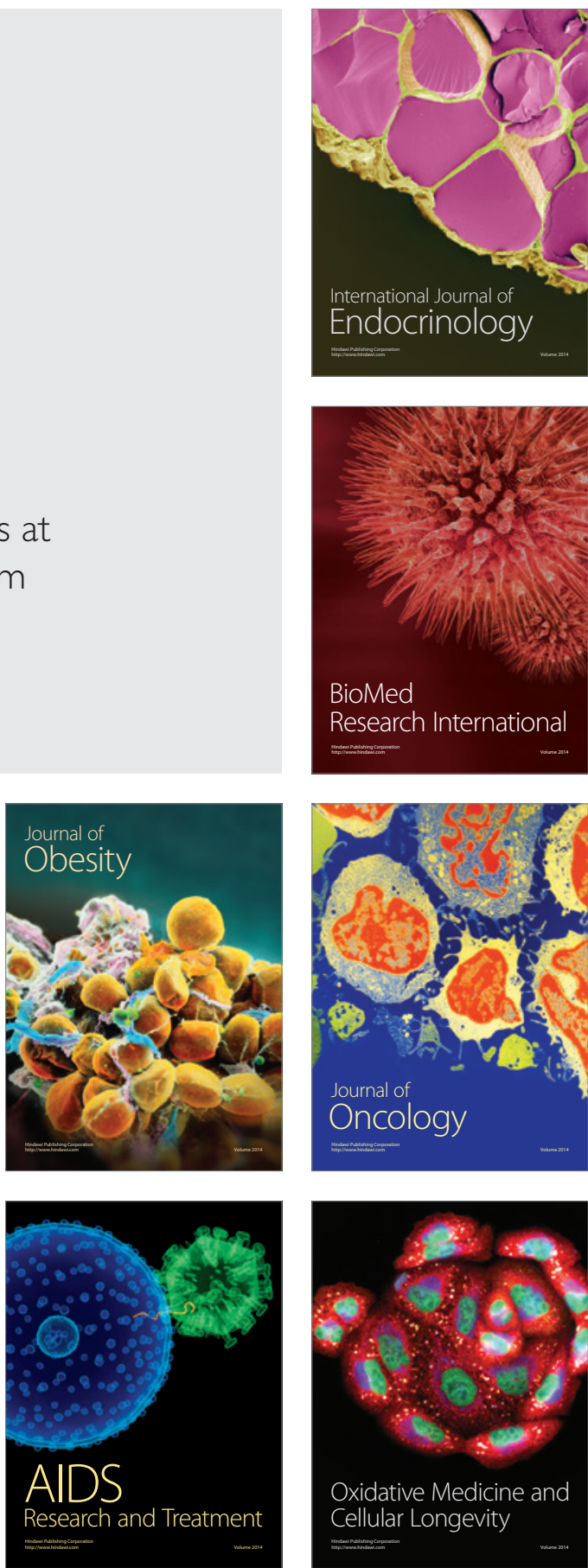